\title{
Three-Dimensional Shape-Motion Analysis of the Left Anterior Descending Coronary Artery in EBCT Images
}

\author{
Ioannis A. Kakadiaris ${ }^{\star}$, Amol Pednekar, and Alberto Santamaría-Pang
}

Visual Computing Lab, Dept. of Computer Science, Univ. of Houston, Houston, TX

\begin{abstract}
In this paper, we present a physics-based deformable model framework for the quantification of shape and motion parameters of the Left Anterior Descending (LAD) coronary artery in the heart's local frame of reference. We define the long-axis of the heart as the local frame of reference. The shape of the LAD is modeled as a parametric curved axis with Frenet-Serret frame. The motion of the LAD (due to heart motion) is modeled as a composite of primitive shape-motion components: 1) longitudinal elongation, 2) radial displacement, and 3) twist with respect to the heart's local frame of reference. The three-dimensional shape-motion components are parameterized along the LAD's length. Results from simulated data and three asymptomatic subjects' Electron Beam Computed Tomography (EBCT) data are in agreement with the expected physiological trends.
\end{abstract}

\section{Introduction}

The significant increase in spatio-temporal resolution of computed tomography (CT) and magnetic resonance (MR) imaging has made it possible to acquire high-resolution volumetric cardiac image data over the cardiac cycle, thus enabling computer-assisted physiological analysis of the heart for diagnosis, prognosis, treatment planning, and monitoring [1. The quantitative analysis of the volume, shape, deformation, and motion of the heart can help differentiate between normal and pathological hearts. The motion and deformation of the heart can be analyzed either by tracking the myocardium or by tracking anatomical, implanted, or induced landmarks. The complexity of the left ventricle (LV) nonrigid motion and the lack of reference landmarks within the myocardium make it difficult to extract the true motion trajectories of tissues. The LAD attached to the myocardium can be used as a natural landmark for tracking anterior-septal myocardial motion.

Previous approaches towards tracking of coronary arteries can broadly be classified as: 1) landmark-based [2314], 2) template-based [5], and 3) geometric

\footnotetext{
* This material is based upon work supported in part by the National Science Foundation under Grants IIS-9985482 and IIS-0335578. Any opinions, findings, and conclusions or recommendations expressed in this material are those of the authors and do not necessarily reflect the views of the National Science Foundation.
} 
constraint-based methods $6,7,8]$. Early coronary artery motion studies followed a landmark-based approach. Later, entire vessel analysis was performed using individual two-dimensional projections. Recent methods track the three-dimensional reconstruction of the arteries [6]. However, these methods do not accommodate radial displacement and axial torsion, as these parameters cannot be derived in a vessel's local frame of reference. This paper presents an extension of our previous work [9] in a physics-based deformable model framework for a clinically relevant parametric shape-motion analysis of the LAD. This new approach takes into consideration a common frame of reference for each subject by defining a local coordinate system for the heart [10]. Our main contribution is that our framework allows for three basic motion components that naturally describe the deformation of the LV that is imparted onto the LAD. This clinically relevant and anatomically normalized geometric reference frame allows comparison between various subjects.

\section{Shape-Motion Modeling of the LAD}

The shape-motion estimation of a non-rigid object poses challenges in terms of establishing point-to-point correspondences in space and over time. Furthermore, the shape modeling for anatomic structure poses the challenge of capturing the clinically relevant shape features along with the features' spatial variance [1], while motion modeling requires combining the clinical significance with the topological interdependence and spatial variance of the motion features. Thus, anatomy related shape-motion modeling requires: 1) an anatomy-relevant local frame of reference; 2) parameterization along the direction of the salient feature's spatial variation; and 3) decomposition of overall motion to capture interdependence of anatomical elements. To that effect, a physics-based deformable model of the LAD is used, where global deformation parameters represent the salient features of the shape changes and motion imparted by the myocardium onto the LAD. Then, shape-motion estimation for the LAD is performed using frame-by-frame tracking of the LAD over time. In this section, we provide a brief description of the deformable model framework [12,13], and its adaptation for shape-motion analysis of the LAD.

\subsection{Model-Centered Frame of Reference}

The definition of an object's own local coordinate system, effectively capturing its shape and motion specific features, is essential for the analysis of the shape, motion, and deformation of the object. Thus, determining the heart's local coordinate system is important for establishing the topological relationship between the heart and the coronary arteries. The local heart coordinate system enables the characterization of the coronary artery motion in terms of components relative to the medial axis of the heart.

Geometrically our LAD model consists of a 3D surface in space with intrinsic material coordinates, $\mathbf{u}=(u, v)$. The positions of the points on the model relative 
to an inertial frame of reference $\Phi$ in space are given by a vector-valued, timevarying function of $\mathbf{u}: \mathbf{x}(\mathbf{u}, t)=\left(x_{1}(\mathbf{u}, t), x_{2}(\mathbf{u}, t), x_{3}(\mathbf{u}, t)\right)^{\top}$, where ${ }^{\top}$ denotes transposition. A non-inertial, model-centered reference frame $\phi$ is set up and the position function is expressed as: $\mathbf{x}=\mathbf{c}+\mathbf{R} \mathbf{p}$, where $\mathbf{c}(t)$ is the origin of $\phi$ at the center of the model and the rotation matrix $\mathbf{R}(t)$ gives the orientation of $\phi$ relative to $\Phi$. Thus, $\mathbf{p}(\mathbf{u}, t)$ gives the positions of points on the model relative to the model frame.

We define the heart's coordinate system with its origin at the center of the base of the heart. The longitudinal axis of the heart's coordinate system is defined as the line between the midpoint of the split between ascending and descending pulmonary trunk and the LV apex (Figs. 1 (a,b)). Thus, orientation and position of the long axis (with respect to the measured trans-axial images) are defined by two rotation angles and the origin's 3D coordinates, which are used to re-orient the trans-axial data to the local coordinates of the heart.

\subsection{Parametric Shape Model}

To incorporate global and local deformations, $\mathbf{p}$ is expressed as the sum of a global reference shape $\mathbf{s}(\mathbf{u})$ and a local displacement function $\mathbf{d}(\mathbf{u}, t): \mathbf{p}=\mathbf{s}+\mathbf{d}$. The geometric model of the LAD is a tube-shaped parametric deformable model with a curved axis $\mathbf{e}(u)$ and Frenet-Serret (FS) frame oriented cross sectional planes $\mathbf{a}(\mathbf{u})$, defined as follows:

$$
\mathbf{s}(u, v)=\left[\begin{array}{l}
e_{1}(u)+a_{1}(u, v) \\
e_{2}(u)+a_{2}(u, v) \\
e_{3}(u)+a_{3}(u, v)
\end{array}\right]
$$

where $-\frac{\pi}{2} \leq u \leq \frac{\pi}{2},-\pi \leq v \leq \pi$. The parametric curved axis is expressed as a $9^{t h}$-degree polynomial to model the medial axis of the LAD. The degree of the polynomial was selected in order to capture the global shape of the tortuous medial axis of the LAD. The FS frame is used to capture the LAD along the medial axis since it provides a local frame of reference uniquely determined by a point on a curve and the curve's behavior around this point. A FS frame is independent of the curve's coordinate system, and the parameterization depends only on its local shape. The orthogonal system of axes that constitutes the FS frame is obtained from the axis derivatives:

$$
\mathbf{T}(u)=\frac{\dot{\mathbf{e}}(u)}{|\dot{\mathbf{e}}(u)|}, \quad \mathbf{B}(u)=\frac{\dot{\mathbf{e}}(u) \times \ddot{\mathbf{e}}(u)}{|\dot{\mathbf{e}}(u) \times \ddot{\mathbf{e}}(u)|}, \quad \mathbf{N}(u)=\mathbf{B}(u) \times \mathbf{T}(u) .
$$

Thus, the cross sectional planes can be represented in a matrix form as follows:

$$
\left[\begin{array}{l}
a_{1}(u, v) \\
a_{2}(u, v) \\
a_{3}(u, v)
\end{array}\right]=r(u)\left[\begin{array}{ll}
N_{1}(u) & B_{1}(u) \\
N_{2}(u) & B_{2}(u) \\
N_{3}(u) & B_{3}(u)
\end{array}\right]\left[\begin{array}{l}
\cos (v) \\
\sin (v)
\end{array}\right]
$$

where $r(u)$ is a radius of the circular cross section. Figure 1(c) illustrates the FS frames of an axis $\mathbf{e}(u)$ for various values of the parameter $u$. 


\subsection{Parametric Shape-Motion Estimation}

The degrees of freedom of the model are incorporated into the vector $\mathbf{q}=$ $\left(\mathbf{q}_{c}^{\top}, \mathbf{q}_{\theta}^{\top}, \mathbf{q}_{s}^{\top}, \mathbf{q}_{d}^{\top}\right)^{\top}$, which consists of the parameters necessary to define the translation $\mathbf{q}_{c}$, rotation $\mathbf{q}_{\theta}$, global deformation $\mathbf{q}_{s}$, and local deformation $\mathbf{q}_{d}$ of the model 1314. The aim here is to extract only the global shape change and motion imparted by myocardium onto the LAD. To that end, the degrees of freedom of the model are restricted to global deformation only. The motion of the LAD (due to the heart motion) is modeled as a composite sequence of primitive shape-motion components: 1) longitudinal elongation $\left(\mathbf{T}_{\mathbf{L E}}\right)$ along the length of the LAD; 2 ) radial displacement $\left(\mathbf{T}_{\mathbf{R D}}\right)$ with respect to the long axis of the heart; and 3 ) twist $\left(\mathbf{T}_{\mathbf{T}}\right)$ around the long axis of the heart. The axis of the LAD is initialized as the shape and position of the LAD over time, given by:

$$
\mathbf{m}(u, v, t)=\mathbf{T}_{\mathbf{T}}(u, t) \cdot\left(\mathbf{T}_{\mathbf{R D}}(u, t) \cdot\left(\mathbf{T}_{\mathbf{L E}}(u, t) \cdot(\mathbf{s}(u, v))\right)\right)^{\top},
$$

where $\mathbf{m}(u, v, t)=\left(m_{1}(u, v, t), m_{2}(u, v, t), m_{3}(u, v, t)\right)^{\top}$ and $\mathbf{m}(u, v, 0)=\mathbf{s}(u, v)$. The longitudinal elongation (or shrinking) along the length of the artery can be parameterized as follows:

$$
\mathbf{T}_{\mathbf{L E}}(u, t)=\left[1,1, c^{l}(u, t)\right]^{\top},
$$

where $c^{l}(u, t)$ is a time-varying longitudinal elongation parameter function. The radial displacement with respect to the long axis of the heart can be parameterized as follows: $\mathbf{T}_{\mathbf{R D}}(u, t)=\left[c_{1}^{r}(u, t), c_{2}^{r}(u, t), 1\right]^{\top}$, where $c_{1}^{r}(u, t)$ and $c_{2}^{r}(u, t)$ are the time-varying radial displacement parameter functions. Finally, the twist around the long axis of the heart can be parameterized as follows:

$$
\mathbf{T}_{\mathbf{T}}(u, t)=\left[\begin{array}{ccc}
\cos \left(c^{t}(u, t)\right) & -\sin \left(c^{t}(u, t)\right) & 0 \\
\sin \left(c^{t}(u, t)\right) & \cos \left(c^{t}(u, t)\right) & 0 \\
0 & 0 & 1
\end{array}\right],
$$

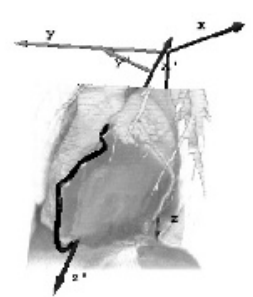

(a)

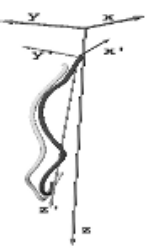

(b)

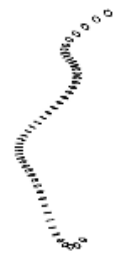

(c)

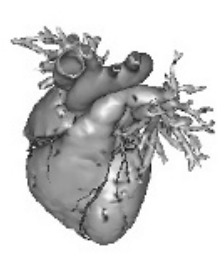

(d)

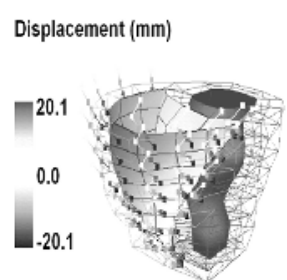

(e)

Fig. 1. (a) Local coordinate system of the heart. (b) Segmented LAD at end-systole (ES) and end-diastole (ED) and heart-centered coordinate system. (c) Shape model of the LAD with FS frames. (d) A geometric model of the heart from Visible Productions $^{\text {TM }}$. (e) A geometric model and motion data of a human subject's ventricles extracted using MRI-SPAMM data [1516] (Courtesy: Prof. Dimitri Metaxas). 
where $c^{t}(u, t)$ is the time-varying twisting parameter function along the LAD's length. Thus, the vector of motion parameters is defined as: $\mathbf{q}_{m}=\left(c^{l}, c_{1}^{r}, c_{2}^{r}, c^{t}\right)^{\top}$. Furthermore, the Jacobian matrix is computed as $\mathbf{J}=\frac{\partial \mathbf{m}}{\partial \mathbf{q}_{\mathbf{m}}}$, in order to formulate the dynamics of the deformable model through the application of Lagrangian mechanics. The resulting Lagrangian equations for the LAD shape-motion estimation task are of the form $\dot{\mathbf{q}}_{m}+\mathbf{K} \mathbf{q}_{m}=\mathbf{f}_{q}$ where $\mathbf{K}$ is the stiffness matrix of the simulated elastic material [14], and the vector $\mathbf{f}_{q}$ represents the external forces that the LAD data apply to the model. Fitting is accomplished by integrating the Lagrangian equations through time using the physics-based modeling framework [1213]. Once the equilibrium is reached, the values of $\mathbf{q}_{m}$ are the shape-motion parameters of the LAD.

\subsection{Algorithm}

The LAD tracking is comprised of the following three main steps:

Step 1 - Determine the local coordinate system of the heart: The long-axis of the heart is localized manually by selecting the center of the base and the apex of the heart. The splitting of the pulmonary trunk occurs two or three slices above the base of the heart (where the left main coronary artery starts) and serves as a landmark to locate the center of the base of the conical heart. The apex of the conical heart is localized about 6-8 slices below the first appearance of the liver in the EBCT data.

Step 2 - Fitting the data at the end-systole (ES): The parametric medial axis $\mathbf{e}(u)$ is computed from the center points corresponding to the LAD's 3D contours (currently, determined manually in each phase). Then, the deformablemodel is initialized as: $\mathbf{m}(u, v, 0)=\mathbf{s}(u, v)$ to fit the LAD at ES [14.

Step 3 - Fitting of a deformable model from phase to phase: Global deformations are used to deform the fitted model at the ES from phase to phase, producing a vector of motion parameters $\mathbf{q}_{m}$ at every phase. Global deformations are computed from the corresponding LAD's 3D contours of each phase.

\subsection{Experimental Data}

We have performed a number of experiments to: 1) assess the validity of our LAD shape-motion estimation model, and 2) compare the range of shape-motion parameters computed by our method with the torsional measurements reported for LV through physiological experiments.

To simulate the LAD deformation over the cardiac cycle, we considered a prototype consisting of the following two components: 1) a geometric model of the heart from Visible Productions ${ }^{\mathrm{TM}}$; these geometric models have been extracted using the Visible Human Data and include both the heart surface and the coronary artery tree (Fig. 1)(d)); 2) the patient-specific ventricles motion data provided by Prof. Metaxas (Rutgers University); these motion data were obtained using the techniques detailed in 1516] (Fig.11(e)). The geometric model was animated using the patient-specific motion data from the LV and right ventricle $(\mathrm{RV})$. To study the LAD motion on real data, three volunteer subjects' 
EBCT images were acquired on a GE Imatron EBCT scanner. The acquisition parameters were: resolution $0.41 \times 0.41 \times 1.50 \mathrm{~mm} ; 5$ phases from ES to end-diastole (ED); 82 slices per phase, with presence of contrast agent.

\section{Results and Discussion}

Due to space limitation we present the estimated shape-motion parameters $\left(\mathbf{T}_{\mathbf{T}}\right.$, $\mathbf{T}_{\mathbf{L E}}$, and $\mathbf{T}_{\mathbf{R D}}$ ) of the LAD for the simulated data and for Subject-3. Figures 2 $2(a, b)$ shows the twist angles for simulated data and Subject-3 around the long-axis of the heart. Positive twist angle represents counter-clockwise circumferential motion when looking from apex towards base from ES to ED. The twist angles for simulated data at the ED vary from $-3.26^{\circ}$ in the basal third to $-0.34^{\circ}$ in the apical third, taking a minimum value of $-22.67^{\circ}$ in the apical third. While for Subject-3, twist angles at the ED phase range from $0.94^{\circ}$ in the basal third to $3.56^{\circ}$ in the apical third, with a minimum value of $-13.97^{\circ}$ at the basal third. In both cases, the magnitude of torsional deformation increases from the base towards the apex. For Subject-3, an untwisting effect at the apex can be observed. It is important to mention that for Subject-3 the LAD's apical segment covers a longer portion of the apex compared to that of the simulated data, resulting in positive twist values for Subject-3 when the apex moves from ES to ED. These values and torsion patterns are consistent with those computed using biplane cineradiography of tantalum helices implanted within the LV midwall [17. Figures 2(c-h) depict the estimated shape-motion parameters for Subject-3 with their corresponding rate of change. Positive-negative values for $\mathbf{T}_{\mathbf{L E}}$, and $\mathbf{T}_{\mathbf{R D}}$ represent longitudinal elongation-contraction and radial displacement away-towards the long-axis of the heart. The mean and standard deviation values per segment from ES to ED for $\mathbf{T}_{\mathbf{T}}, \mathbf{T}_{\mathbf{L E}}$, and $\mathbf{T}_{\mathbf{R D}}$ are: $\left(-3.87^{\circ} \pm 6.76^{\circ}\right),(3.56 \mathrm{~mm} \pm 2.71 \mathrm{~mm})$, and $(0.63 \mathrm{~mm} \pm 1.43 \mathrm{~mm})$ respectively.

Table 1 shows the mean, standard deviation, maximum, and minimum values for the shape-motion parameters in the basal, mid-ventricular, and apical segments for Subject-3. The $\mathbf{T}_{\mathbf{R D}}$ values suggest that the overall LAD moves away from the long-axis of the heart. $\mathbf{T}_{\mathbf{R D}}$ shows higher values in the basal segment, decreasing in the apical segment, moving towards the long-axis of the heart at the extreme apical segment. The increasing values of $\mathbf{T}_{\mathbf{L E}}$ from base to apex indicate that the LAD's elongation takes place primarily from the mid-ventricular to the apical segment. These shape-motion parameters along with the rate of change from ES to ED, show physiological trends expected for asymptomatic hearts [10].

Our model provides a clinically relevant and anatomically normalized geometric reference frame, which allows comparison between various subjects. This suggests the possibility of classifying parameters associated with normal and pathological hearts. This model would serve as a detailed morphological model describing the interaction between coronary fluid flow and the vessel wall for the computational fluid dynamics simulations that will elucidate the biomechanical implications of the coronary arterial motion in vascular disease. The preoperative 


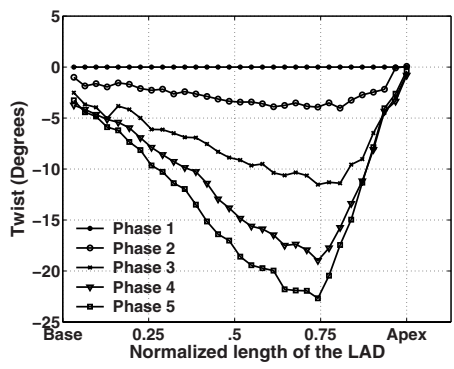

(a)

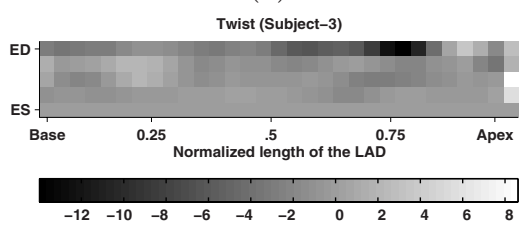

(c)

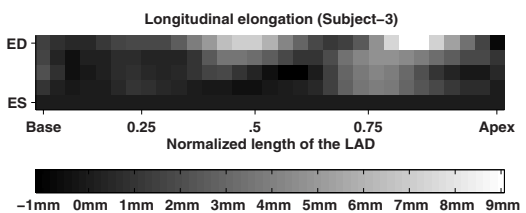

(e)

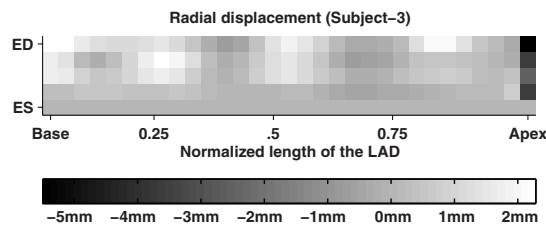

(g)

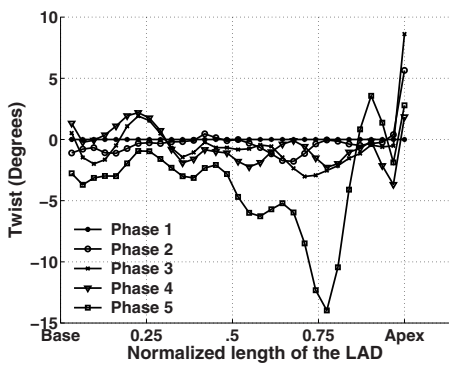

(b)

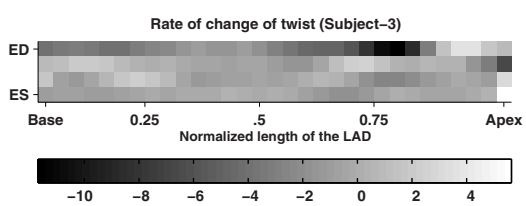

(d)

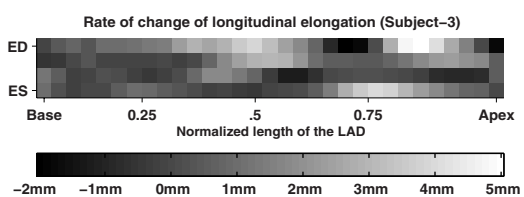

(f)

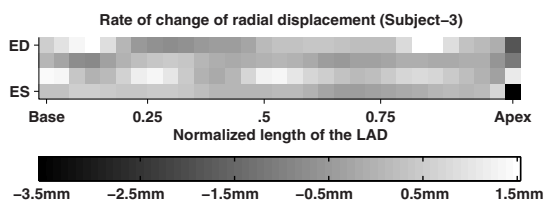

(h)

Fig. 2. Twist parameters from ES to ED for: (a) simulated data, and (b) Subject3. Estimated parameters (Subject-3) for: (c) twist, (d) rate of change of twist, (e) longitudinal elongation, (f) rate of change of the longitudinal elongation, (g) radial displacement, and $(\mathrm{h})$ rate of change of the radial displacement.

Table 1. Statistics corresponding to Subject-3's shape-motion parameters (ED) at three sections of the LAD: basal, mid-ventricular, and apical.

\begin{tabular}{|l|r|c|r|r|r|r|r|r|r|r|r|r|}
\hline & \multicolumn{4}{|c|}{ Basal } & \multicolumn{4}{c|}{ Mid } & \multicolumn{4}{c|}{ Apical } \\
\cline { 2 - 12 } & mean & std & max & min & mean & std & max & min & mean & std & max & \multicolumn{1}{c|}{ min } \\
\hline $\mathbf{T}_{\mathbf{T}}$ & -2.33 & 0.94 & -0.94 & -3.70 & -4.12 & 1.61 & -2.08 & -6.27 & -4.42 & 6.26 & 3.56 & -13.97 \\
$\mathbf{T}_{\mathbf{R D}}$ & 1.40 & 0.54 & 2.29 & 0.50 & 0.44 & 0.90 & 1.83 & -0.65 & 0.10 & 2.06 & 2.09 & -5.50 \\
$\mathbf{T}_{\mathbf{L E}}$ & 1.41 & 0.69 & 2.65 & 0.45 & 4.53 & 2.14 & 7.07 & 1.09 & 4.64 & 3.27 & 9.07 & -0.74 \\
\hline
\end{tabular}


patient-specific shape-motion parameters can be used for robotic surgery on the beating heart. Also, it can be used to compute geometric features like curvature, torsion, and discrete flexion points in the LAD's local frame of reference.

\section{Conclusion}

The model-based technique proposed provides a framework to extract clinically relevant shape-motion parameters of the LAD along its entire length for all the phases of the cardiac cycle.

\section{References}

1. Frangi, A., Niessen, W., Viergever, M.: Three-dimensional modeling for functional analysis of cardiac images: a review. IEEE TMI 20 (2001) 2-25

2. Kong, Y., Morris, J.J., McIntosh, H.D.: Assessment of regional myocardial performance from biplane coronary cineangiograms. Am. Cardiology 27 (1971) 529-537

3. Potel, M.J., Rubin, J.M., MacKay, S.A., Aisen, A.M., Al-Sadir, J.: Methods for evaluating cardiac wall motion in three-dimensions using bifurcation points of the coronary arterial tree. Investigative Radiology 18 (1983) 47-57

4. Stevenson, D.J., Smith, I., Robinson, G.: Working towards the automated detection of blood vessels in X-ray angiograms. Pattern Recognition Letters 2 (1987) 107-112

5. Ding, Z., Friedman, M.: Quantification of 3-D coronary arterial motion using clinical biplane cineangiograms. The Int. J. of Card. Imag. 16 (2000) 331-346

6. Chen, S., Carroll, J.: Kinematic and deformation analysis of 4-D coronary arterial trees reconstructed from cine angiograms. IEEE TMI 22 (2003) 710-721

7. Olszewski, M., Long, R., Mitchell, S., Wahle, A., Sonka, M.: A quantitative study of coronary vasculature in four dimensions. In: Proceedings of the 22nd Annual International Conference of the IEEE EMBS. Volume 4. (2000) 2621-2624

8. Liao, R., Chen, S.J., Messenger, J., Groves, B., Burchenal, J., Carroll, J.: Fourdimensional analysis of cyclic changes in coronary artery shape. Catheterization and Cardiovascular Interventions 55 (2002) 344-354

9. Kakadiaris, I., Pednekar, A., Zouridakis, G., Grigoriadis, K.: Estimating the motion of the LAD: A simulation-based study. In: MICCAI'01. Volume LNCS 2208., Utrecht, The Netherlands (2001) 1328-1331

10. Kwok, L., Miller, D.: Torsional deformation of the left ventricle. The Journal of the Heart Valve Disease 4 (1995) S214-222

11. Frangi, A., Niessen, W., Nederkoorn, P., Bakker, J., Mali, W., Viergever, M.: Quantitative analysis of vascular morphology from $3 \mathrm{D}$ MR angiograms: in vitro and in vivo results. Magnetic Resonance in Medicine 45 (2001) 311-322

12. Terzopoulos, D., Metaxas, D.: Dynamic 3D models with local and global deformations: Deformable superquadrics. IEEE Transactions on Pattern Analysis and Machine Intelligence 13 (1991) 703-714

13. Metaxas, D., Terzopoulos, D.: Shape and nonrigid motion estimation through physics-based synthesis. IEEE T-PAMI 15 (1993) 580-591

14. Metaxas, D., Kakadiaris, I.: Elastically adaptive deformable models. IEEE Transactions on Pattern Analysis and Machine Intelligence 24 (2002) 1310-1321

15. Park, J., Metaxas, D., Young, A., Axel, L.: Deformable models with parameter functions for cardiac motion analysis from tagged MRI data. IEEE Trans. Medical Imaging 15 (1996) 278-289 
16. Haber, E., Metaxas, D., Axel, L.: Motion analysis of the right ventricle from MRI images. In: MICCAI'98. Number 1496, Cambridge, MA (1998) 177-188

17. Hansen, D., Daughters, G., Alderman, E., Ingels, N., Miller, D.: Torsional deformation of the left ventricular midwall in human hearts with intramyocardial markers: regional heterogeneity and sensitivity to the inotropic effects of abrupt rate changes. Circulation Research 62 (1988) 941-952 